\title{
Editorial
}

\section{Modelling and Simulation in Operations and Complex Supply Chains}

\author{
Salvatore Cannella, ${ }^{1,2}$ Rosa G. González-Ramírez, ${ }^{3}$ Roberto Dominguez, ${ }^{4}$ \\ Mónica A. López-Campos, ${ }^{5}$ and Pablo A. Miranda ${ }^{6}$ \\ ${ }^{1}$ DICAR, University of Catania, Catania, Italy \\ ${ }^{2}$ Industrial Management \& Business Administration Department, School of Engineering, University of Seville, Seville, Spain \\ ${ }^{3}$ Universidad de los Andes, Santiago de Chile, Chile \\ ${ }^{4}$ Centre for Management Studies, Instituto Superior Técnico, Technical University of Lisbon, Lisbon, Portugal \\ ${ }^{5}$ Industrial Engineering Department, Universidad Técnica Federico Santa María, Valparaíso, Chile \\ ${ }^{6}$ School of Industrial Engineering, Pontificia Universidad Católica de Valparaíso, Valparaíso, Chile
}

Correspondence should be addressed to Salvatore Cannella; cannella@us.es

Received 29 December 2016; Accepted 29 December 2016; Published 19 January 2017

Copyright (C) 2017 Salvatore Cannella et al. This is an open access article distributed under the Creative Commons Attribution License, which permits unrestricted use, distribution, and reproduction in any medium, provided the original work is properly cited.

As manufacturing practice shifts toward the outsourcing paradigm, the Supply Chain Management (SCM) takes place throughout a high number of entities, thus generating significant interdependence among them. Modelling such complex systems by classic analytic methods represents a challenge since they are characterized by many actors assuming different roles, acting simultaneously and continuously, and reacting to the actions of other agents with a nonpredetermined order. To overcome the inconveniences and limitations of the analytic methods, simulation has been broadly used in modelling and evaluating a wide range of different strategies in SCM, as well as being a decisionmaking tool to improve supply chain performance. However, there is still room for gaining further insights into a wide variety of topics concerning operations and SCM analysis via what-if analysis. In this special volume we bring together 15 high-quality researches (out of 59 submissions) in the modelling and performance evaluation of innovative supply and production networks. Each submission was reviewed by a double-blind review process using at least two reviewers who are experts in the corresponding field. We briefly describe these contributions below.

C. Wang et al. in their paper "Comparison of DualChannel Supply Chain Structures: E-Commerce Platform as Different Roles" provide the performance comparisons of supply chain members between the two different dualchannel supply chain structures and the single channel structure. They use the game theory approach to develop two dual-channel supply chain structures where the e-commerce owner can act as an e-tailer (E Structure) or an agent authorizing the manufacturer to sell products on the platform directly (P Structure). They show that both structures can increase the consumer surplus and social welfare.

R. Ramos-Hernández et al. in their paper "Assessing the Impact of a Vinasse Pilot Plant Scale-Up on the Key Processes of the Ethanol Supply Chain" analyze through various tests and research the impact of the pilot plant's scale-up in the key processes of the company's supply chain. With the help of a sensitivity analysis, this study finds the values that would allow the company to improve its order fulfillment indicator and to increase profits, assuming an expected demand by the introduction of this new product into the market. The results show that (1) the pilot plant only fulfills the $32 \%$ of the orders, (2) according to the current vinasse storage capacity, it is possible to fulfill up to $77 \%$ of the orders by scaling up the pilot plant, (3) satisfying the $100 \%$ of the orders is necessary to use all the vinasse generated, and (4) the highest profit is reached by processing all the vinasse and by considering the upper sale price. 
Y.-S. Liu et al. in their paper "A Composite Contract for Coordinating a Supply Chain with Price and Effort Dependent Stochastic Demand" study (1) how to coordinate the decentralized supply chain and (2) how to determine the optimal sales effort level, pricing, and inventory decisions under the additive demand case. They show, via numerical examples, the effectiveness of combined contract in supply chain coordination and highlight model sensitivities to parametric changes.

S. Maldonado-Pinto et al. in their paper "Analyzing the Performance of a Hybrid Heuristic for Solving a Bilevel Location Problem under Different Approaches to Tackle the Lower Level" present a combinatorial bilevel programming problem. Specifically, they develop a hybrid algorithm for solving a battery of benchmark instances. According to their results the proposed algorithm outperforms the evolutionary algorithm already existing in the literature by including a more sophisticated procedure for improving the population through the generations which accelerates the convergence of the algorithm. Finally they perform a study for illustrating the impact in the algorithm's performance when solving the lower level through three different exact or heuristic approaches.

A. Paz et al. in their paper "Highway Expenditures and Associated Customer Satisfaction: A Case Study" analyze the satisfaction of Nevadans with respect to their highway transportation system and the corresponding expenditures of the Nevada Department of Transportation (NDOT). They design a survey questionnaire to capture the opinions of Nevadans (customers) about a number of characteristics of their transportation system. Their results indicate how the customer satisfaction is decreasing with respect to traffic safety throughout Northwestern and Southern Nevada highways.

H. Zhang and J. Jiang in their paper "Informed Principal Model and Contract in Supply Chain with Demand Disruption Asymmetric Information" present a supply chain with one manufacturer and one retailer, in which the demand of the retailer is uncertain and may suffer supply disruptions.

N. Sáinz Bernat et al. in their paper "Empty Container Management at Ports considering Pollution, Repair Options, and Street-Turns" present several new, inventory based empty container management policies using emissions prices for a cost function that integrates economic and ecological objectives, on the sea and land side. Specifically, they examine the proposed policies analytically and evaluate them in a simulation approach with metaheuristic parameter search, assuming the case of a Latin American shipping service operated by a major shipping company. They find that the presented model may actually enable planners to master the observed potential trade-off in objectives and may be an effective way to more realistic, more sustainable empty container management.

W. Ran et al. in their paper "A Study of the ClosedLoop Supply Chain Coordination on Waste Glass Bottles Recycling" propose a new model to coordinate the profit of manufacturers and retailers in a closed-loop supply chain with revenue sharing contract. They find that an integrated overall recycling strategy (retailer-manufacturer) is better than the classical single node recycling model.

K. E. Hernandez-Ruiz et al. in their paper "Optimizing Safety Stock Levels in Modular Production Systems Using Component Commonality and Group Technology Philosophy: A Study Based on Simulation" develop a mathematical model to reduce safety stock levels in organizations that employ modular production. To construct the model, they take advantage of the benefits of aggregate inventories, standardization of components, component commonality, and Group Technology philosophy in regard to stock levels. They test the proposed model through the simulation of three years of operation of two modular product systems. For each system, they calculate and compare the safety stock levels for two cases: (1) under the only presence of component commonality and (2) under the presence of both component commonality and Group Technology philosophy. Their results show a reduction in safety stock levels when we linked the component commonality with the Group Technology philosophy.

P. Kefer et al. in their paper "Fuzzy Multicriteria ABC Supplier Classification in Global Supply Chain" define the appropriate purchasing strategy in the global supply chain through two steps: (1) classification of a large number of potential suppliers by applying proposed fuzzy multicriteria $\mathrm{ABC}$ and (2) the ranking of classified A group of suppliers (optimal portfolio of suppliers) by using the proposed model consisting of fuzzy AHP and fuzzy ELECTRE methods. The developed two-step model is verified on real life data. Their results indicate good compliance with the opinions management in this type of industry.

Z. Shi et al. in their paper "Reverse Revenue Sharing Contract versus Two-Part Tariff Contract under a ClosedLoop Supply Chain System" study different coordination performance of two types of contracts, two-part tariff (TTC) and reverse revenue sharing contract (RRSC), in a twoechelon CLSC system. Through a mathematical analysis, they find that performance improves by adjusting the ratio of transfer collection price through RRSC. Furthermore, they provide the function to estimate the appropriate ratio of transfer collection price.

G. Deng et al. in their paper "An Enhanced Discrete Artificial Bee Colony Algorithm to Minimize the Total Flow Time in Permutation Flow Shop Scheduling with Limited Buffers" propose a discrete artificial bee colony algorithm (DABC) for solving the permutation flow shop scheduling problem with limited buffers. Their results show how the proposed algorithm is not only superior to the existing discrete differential evolution algorithm and iterated greedy algorithm but also performs better than two recently proposed discrete artificial bee colony algorithms.

H. Du et al. in their paper "A Hybrid Algorithm Based on Particle Swarm Optimization and Artificial Immune for an Assembly Job Shop Scheduling Problem" propose a new particle swarm algorithm based on artificial immune to solve the AJSSP. The complete time minimization is the objective of scheduling. The principle of particle swarm optimization algorithm and steps of proposed HPSO method are described, and hybrid algorithm is applied into a case study 
development from classical FT06. According to the authors' statement the experiment result confirmed its practice and effective.

J. Ma et al. in their paper "Study on Complex Advertising and Price Competition Dual-Channel Supply Chain Models Considering the Overconfidence Manufacturer" develop a dual channel supply chain competition model including two manufacturers and two retailers. Manufacturers who consider advertising as the decision variables have overconfidence behavior; the retailers adjust their decisions according to the retail price of the product and are rick oriented. They find that under the master-slave game model the profit of manufacturers is greater than the one under decentralized decision making, and the profit of retailers under masterslave game model is less than the one under decentralized decision.

Y. Peng et al. in their paper "A Multiperiod Supply Chain Network Design Considering Carbon Emissions" propose a mixed integer linear programming formulation for modelling and solving a multiperiod one-stage supply chain distribution network design problem. The model is aimed at minimizing two objectives, the total supply chain cost and the greenhouse gas emissions generated mainly by transportation and warehousing operations. Their results show how for a certain range the carbon credit price incentivizes the reduction of carbon emissions to the environment.

As guest editors, we are very satisfied with the quality of the papers presented in this issue. These articles present new results that should be useful for both researchers and managers that are facing decisional issues in supply chains and we guess they contribute to the body of knowledge for this crucial aspect of the business. We hope that readers of this special issue will find not only new ideas and algorithms dealing with the difficult applied problems like supply chain design, assignment problems, bilevel programming models, and so forth but also some interesting results related to regression analysis, as well as the modern simulation techniques.

\section{Acknowledgments}

Finally, we would like to thank the authors for contributing with their high-quality manuscripts and all reviewers for providing valuable comments. Finally, we acknowledge the financial support from the Italian Ministry of Education, University and Research (Rita Levi Montalcini fellow), the European Commission/Andalusian Agency of Knowledge (Talentia Marie Skłodowska-Curie Cofund Fellowship), the Spanish Ministry of Economy, Industry and Competitiveness (Juan de la Cierva Fellowship), and Portuguese Foundation for Science and Technology [Grant SFRH/BPD/108491/2015].

Salvatore Cannella

Rosa G. González-Ramírez Roberto Dominguez

Mónica A. López-Campos

Pablo A. Miranda 


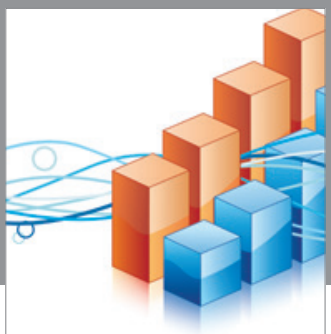

Advances in

Operations Research

vatem alat4

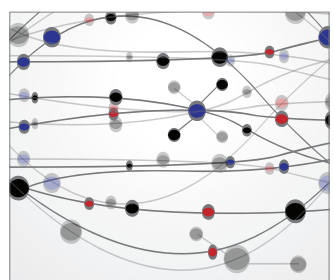

\section{The Scientific} World Journal
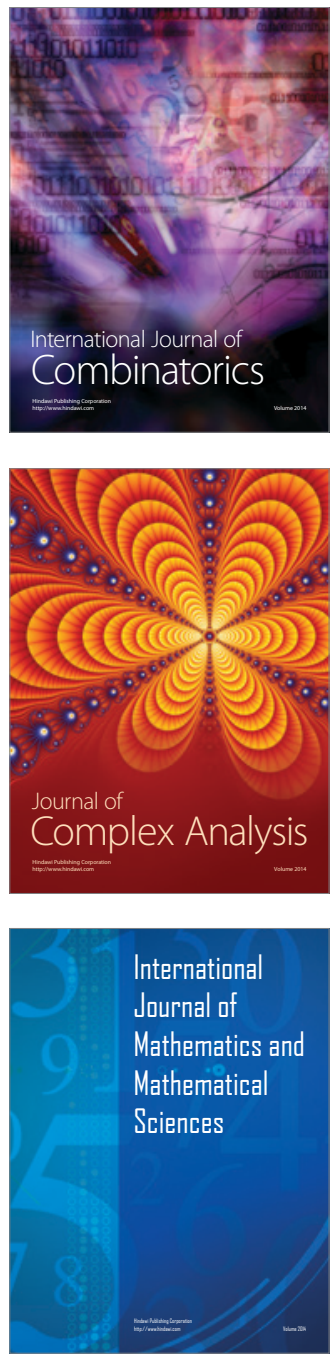
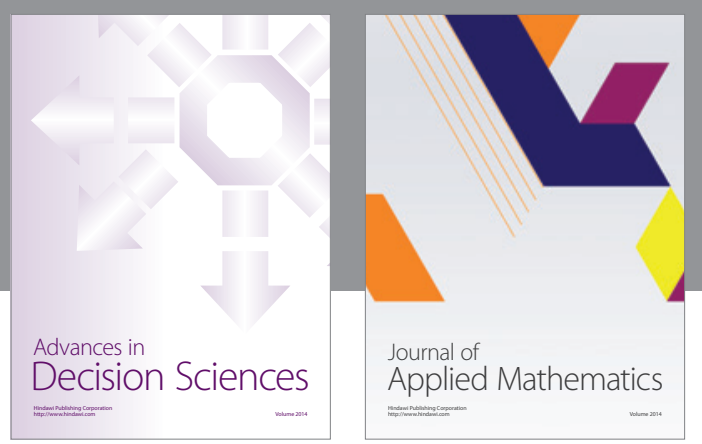

Algebra

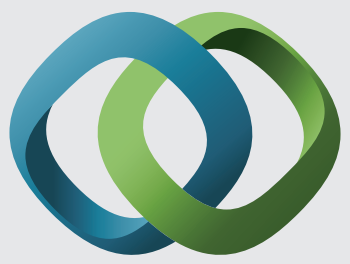

\section{Hindawi}

Submit your manuscripts at

https://www.hindawi.com
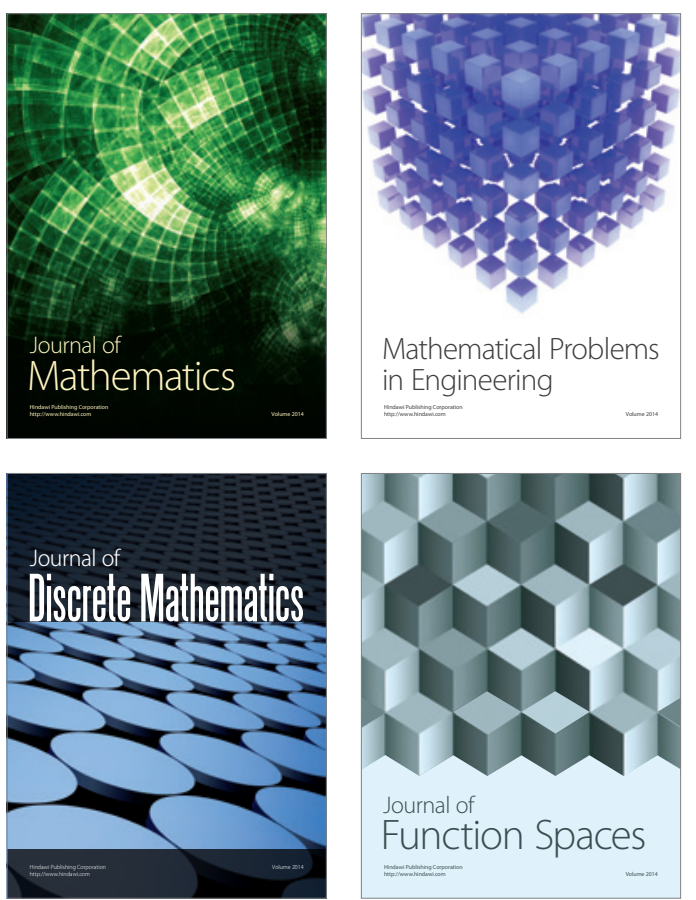

Mathematical Problems in Engineering
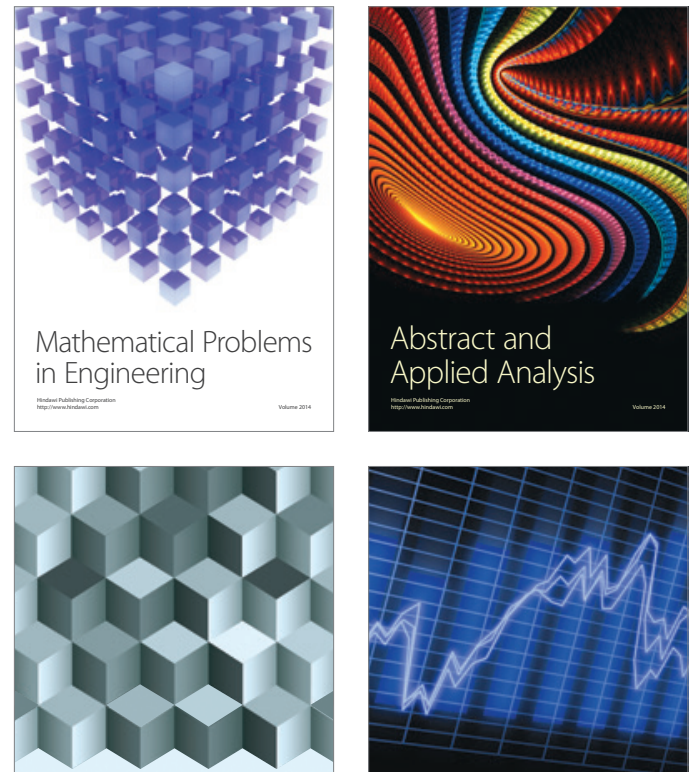

Journal of

Function Spaces

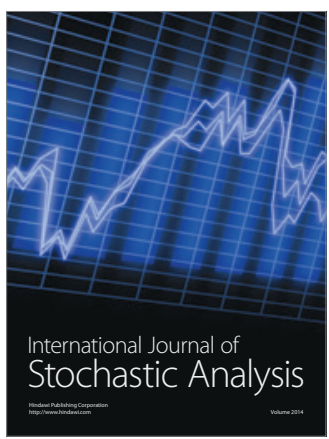

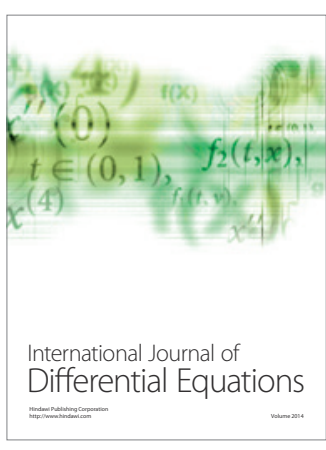
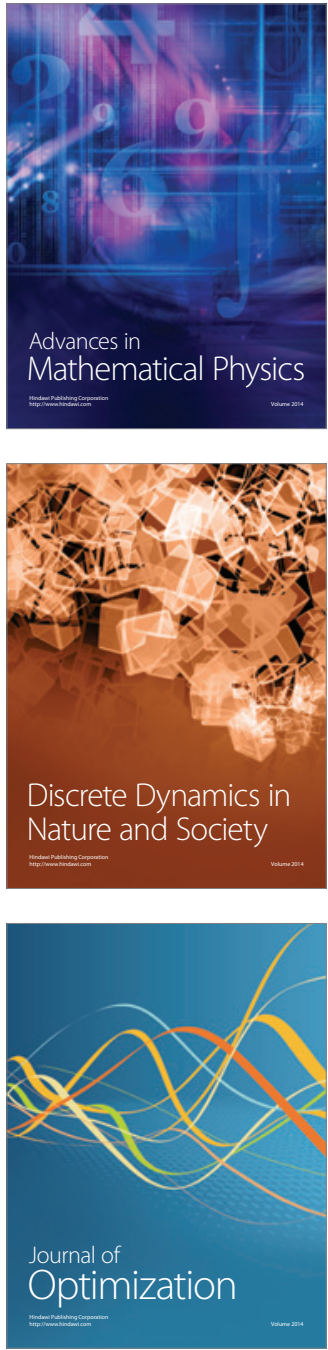